\title{
A Patient with TP53 Germline Mutation Developed Bowen's Disease and Myelodysplastic Syndrome with Myelofibrosis after Chemotherapy against Ovarian Cancer
}

\author{
Kageaki Kuribayashi, Takuya Matsunaga, Toshio SAKaI, Yuko WadA, \\ Kumiko Tateno, Kazuyuki Murase, Akihito FujImi, Rishu TaKimoto, \\ Takeshi Terui, Junji Kato, Aya SASAKI*, Masaaki SATOH* and Yoshiro NiITSU
}

\begin{abstract}
Here we report a case of myelodysplastic syndrome (MDS) with myelofibrosis associated with Bowen's disease. A female patient had undergone an operation and chemotherapy for ovarian cancer when she was 65 years old, and she developed MDS at the age of 70 years old. PCR-single strand conformation polymorphism (SSCP) analysis of peripheral blood mononuclear cells, a Bowen's disease lesion, and normal skin showed an abnormal peak in TP53 exon5. Direct sequencing revealed that they all had missense mutation in codon 175 ( $G$ to $A$ ) of arginine switched to histidine, suggesting a germline mutation of TP53. It was speculated that p53 function was lost by TP53 germline mutation with the loss of a wild type allele induced by the chemotherapy against ovarian cancer, leading to the development of MDS. No therapeutic effects of low dose melphalan or cyclosporine A on MDS were observed, however one month of $30 \mathrm{mg} /$ day prednisolone administration induced a hematological response.
\end{abstract}

(Internal Medicine 44: 490-495, 2005)

Key words: myelodysplastic syndrome, myelofibrosis, Bowen's disease, ovarian cancer, TP53

\section{Introduction}

Myelodysplastic syndrome (MDS) is a clonal hematopoetic stem cell disorder characterized by multi-lineage dysplasia and pancytopenia as a result of ineffective hematopoiesis. One half of the cases show many types of cytogenetic abnormalities, and the disease is heterogeneous not only in its phenotype but also in the molecular mechanism of its genesis and progression (1). TP53 mutations occur in approximately $5 \%$ of MDS cases. As the mutations of TP53 are found early in its diagnosis, it is speculated that TP53 mutations play a role in the development of MDS (2-4). TP53 is located at the short arm of chromosome 17, and their mutations are often associated with $17 \mathrm{p}$ deletion, known as $17 \mathrm{p}$ syndrome in MDS, and acute myeloid leukemia $(1,5)$. These mutations are associated with a typical form of dysgranulopoiesis combining pseudo-Pelger-Huet hypolobulation in neutrophils, which is not apparent in MDS without TP53 mutations.

Here we report a rare case of MDS with myelofibrosis associated with Bowen's disease after surgery and chemotherapy for ovarian cancer. The existence of a TP53 mutation at codon 175 in peripheral blood, the Bowen's disease lesion, and normal skin suggested a germline mutation of TP53.

For editorial comment, see p 406.

\section{Case Report}

A 70-year-old woman was admitted to our hospital with palpitation as the chief complaint in July 2002. She had undergone surgery, followed by chemotherapy using $600 \mathrm{mg}$ docetaxel and $3.0 \mathrm{~g}$ carboplatin for ovarian cancer when she was 65 . Her father had died of carcinoma of the pharynx at 45 years of age and her older brother had died of gastric can-

From the 4th Department of Internal Medicine and *Clinical Pathology, Sapporo Medical University School of Medicine, Sapporo Received for publication August 31, 2004; Accepted for publication December 24, 2004

Reprint requests should be addressed to Dr. Takuya Matsunaga, 4th Department of Internal Medicine, Sapporo Medical University School of Medicine, South-1, West-16, Chuo-ku, Sapporo 060-8543 


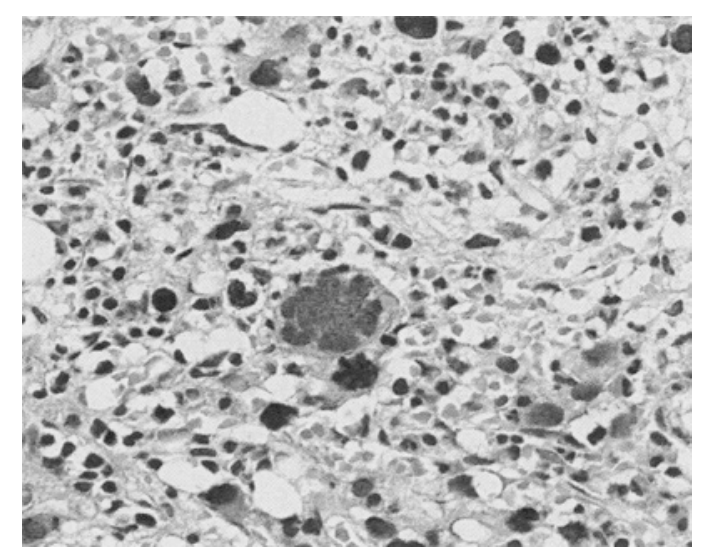

A

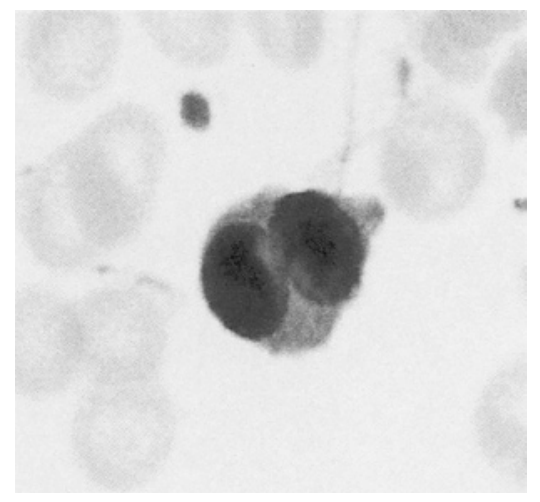

D
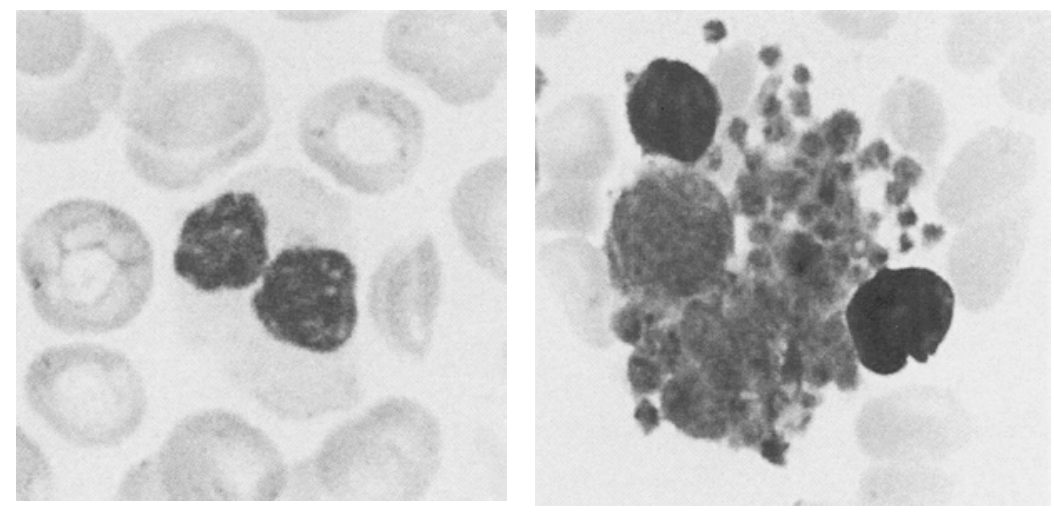

B

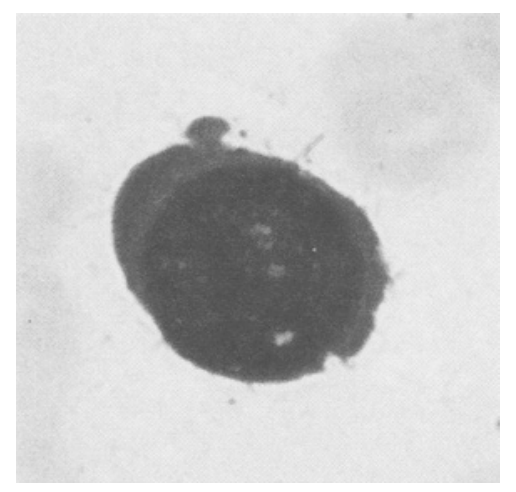

E
C

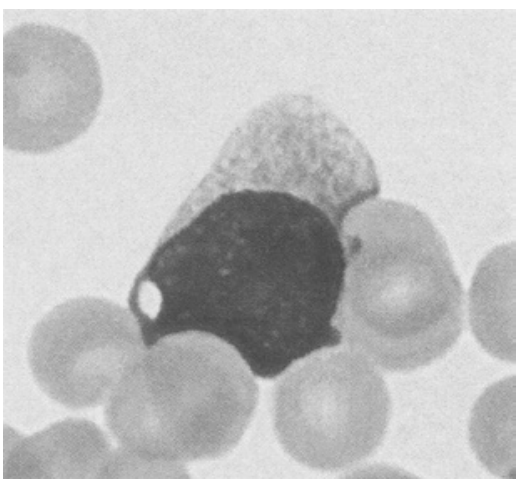

$\mathrm{F}$

Figure 1. HE and Wright-Giemsa staining. A, HE staining of the bone marrow $(\times 200)$. B-E, Wright-Giemsa staining of the bone marrow biopsy touch preparation $(\times 1,000)$. B, Neutrophil with pseudo Pelger-Huet anomaly; C, Giant platelets; D, Erythroblast with multiple nuclei; E, Bone marrow blastic cell; F, Wright-Giemsa staining of peripheral blood blastic cell $(\times 1,000)$.

cer at age 42. Physical examination showed conjunctival pallor and a brownish skin lesion on her back (18 $\mathrm{mm}$ in diameter) with a distinct margin, caused by Bowen's disease. Laboratory investigation showed a decreased leukocyte count $\left(2,000 / \mathrm{mm}^{3} ; 48 \%\right.$ neutrophils, $42 \%$ lymphocytes, $7 \%$ monocytes, $1 \%$ myelocytes, $1 \%$ myeloblasts, $1 \%$ orthochromatic erythrocytes), anemia ( $\mathrm{Hb} 8.6 \mathrm{~g} / \mathrm{dl}$ ), and thrombocytopenia $\left(48,000 / \mathrm{mm}^{3}\right)$. LDH was elevated to $1,821 \mathrm{IU} / \mathrm{ml}$ and LDH-1 and LDH-2 were dominant. Total bilirubin, CEA, CA19-9 and CA125 were within normal limits. Bone marrow aspiration was dry tap. H-E staining of the bone marrow biopsy showed hypercellularity with a marked increase of megakaryocytes (Fig. 1A). May-Giemsa staining of touch preparation of the bone marrow biopsy specimen showed multilineage dysplasia, e.g. dysgranulopoiesis combining neutrophils with pseudo Pelger-Huet anomaly, giant platelets and erythroblasts with multiple nuclei (Fig. 1B, C, D). Azan and Gitter staining showed severe fibrosis of the bone marrow by reticulin and collagen fibers. Cytogenetic analysis using peripheral blood revealed 9 of 15 cells had normal karyotype, although 6 of 15 cells had 44, XX, -5, -6, -7,
$-17,+2$ mar. As her bone marrow aspiration was dry tap, we calculated the percentage of the blasts using touch preparation and it found it to be $12 \%$. The blastic cells of bone marrow (Fig. 1E) and peripheral blood (Fig. 1F) showed round nuclei and basophilic cytoplasm with cytoplasmic blebs. By CD45 blast gating of the peripheral blood, these cells were shown to be positive for CD13, CD33, CD34, CD41, and HLA-DR and were negative for CD3, CD4, CD8, CD10, CD19, and CD20. From these results, the blastic cells were speculated to share myelocytic and megakaryocytic characters. She was diagnosed as having MDS (refractory anemia with excess of blasts) with myelofibrosis.

As she was 70 years old and preferred not to undergo stem cell transplantation, pancytopenia gradually advanced in her clinical course. Two mg/day melphalan was administered from September 16th because it has only mild adverse effects on bone marrow as reported by Denzlinger et al (6). Since the disease progressed even after 2 weeks of melphalan administration, we replaced melphalan with cyclosporine A from November 1st although she had become transfusion dependent to maintain hemoglobin level and 
A

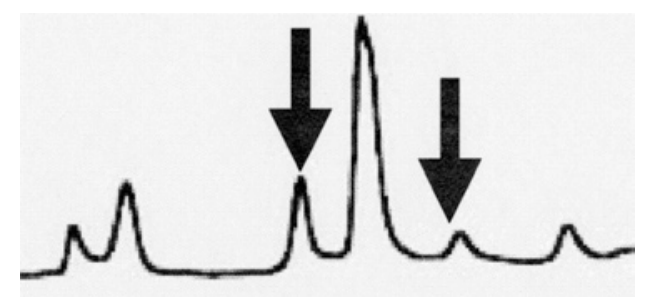

B

C
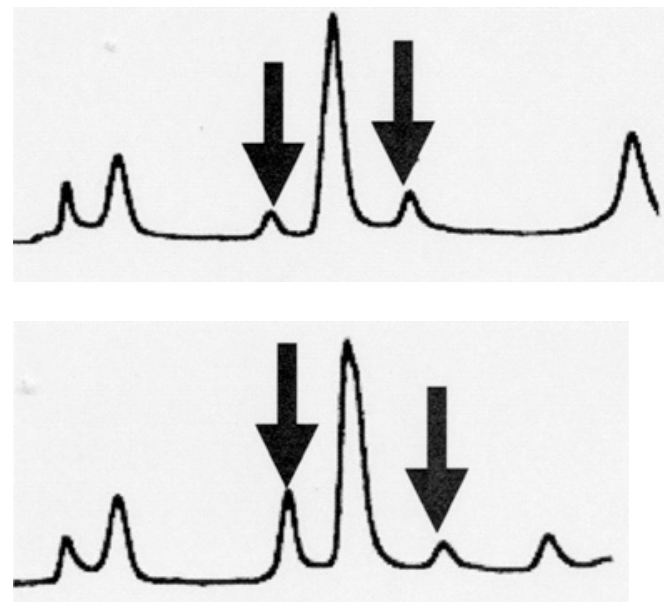

D

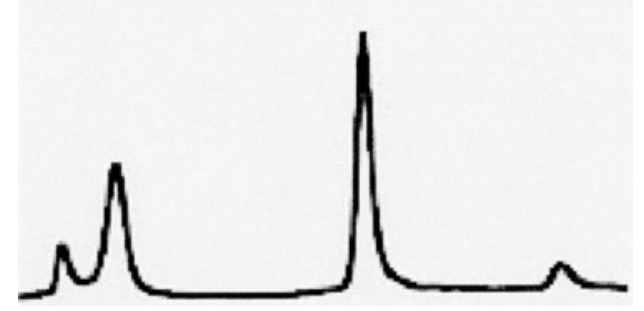

E

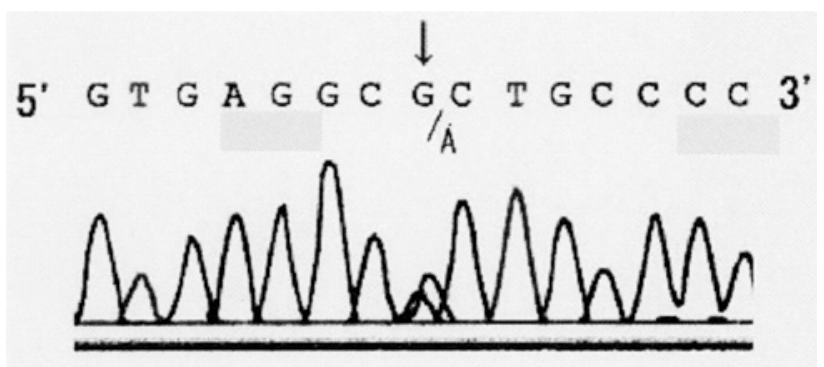

$\mathrm{F}$<smiles>[B]C(C)(C)C=CC</smiles>

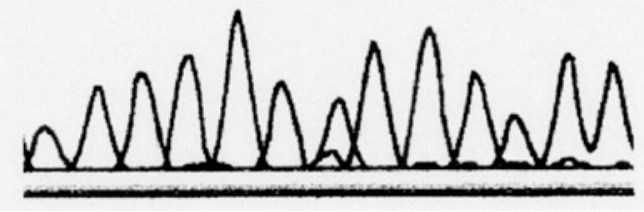

G
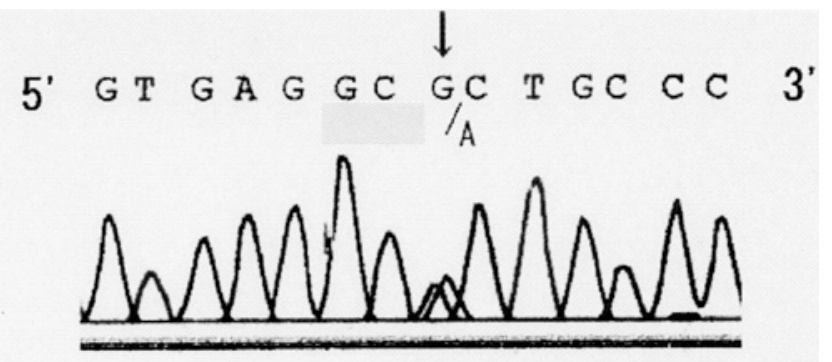

Figure 2. PCR SSCP and sequencing analysis of TP53 exon5. A-D: PCR SSCP analysis of TP53 exon5. A, Peripheral blood; B, Bowen's disease; C, Normal skin; D, Peripheral blood from healthy volunteer. Arrows indicate additional abnormal peak. EG: Direct sequencing analysis of TP53 exon5. E, Peripheral blood; F, Bowen's disease; G, Normal skin. Arrows indicate guanine mutated to adenine in codon 175.

platelet count. From December, she became refractory to transfusion due to association of anti-HLA antibodies and Coombs negative autoimmune hemolysis. Cyclosporine A therapy was stopped at that time, and $30 \mathrm{mg} /$ day of prednisolone was begun from December 31st. The patient gradually recovered from pancytopenia, and a month later she became independent of platelet transfusion, and red blood cell transfusion was reduced from 3 times a week to once a week. From February 1st, we started to reduce prednisolone by $5 \mathrm{mg} /$ week, and she was discharged from our hospital on March 20th, 2003.

Since Kita-Sasai et al (7) and Pedersen-Bjergaard et al (8) have reported that the mutation of TP53 is a poor prognostic factor for MDS, mononuclear cells from peripheral blood were obtained after informed consent, and exons 5-8 of TP53 were analyzed by PCR-SSCP. As shown in Fig. 2A, there was an abnormal peak in exon 5 , which indicates a TP53 mutation.

As TP53 mutations or p53 overexpression occurs in 31 to $47 \%$ of Bowen's disease lesions (9-12), her skin lesion and normal skin was biopsied after informed consent and subjected to immunostaining using the anti-p53 antibody. As shown in Fig. 3A, normal skin showed no staining whereas p53 accumulation in the nuclei was detected in the lesion (Fig. 3B), suggesting the loss of p53 function at the site.

As both peripheral blood and the Bowen's disease lesion in this patient showed TP53 or p53 abnormality, we considered the possibility that this abnormality was hereditary. We therefore extracted DNA from the Bowen's disease lesion and from normal skin. Exons 5-8 of TP53 were analyzed by PCR-SSCP, and it was found that both the Bowen's disease lesion and normal skin showed an abnormal peak in exon5 


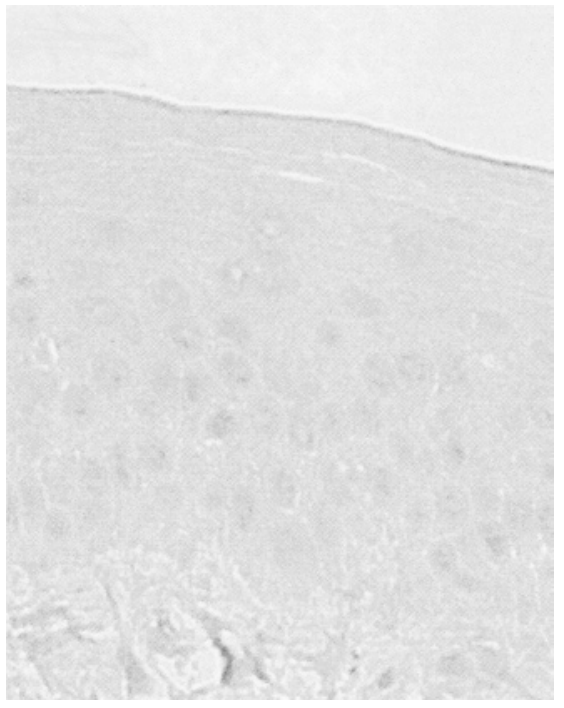

A

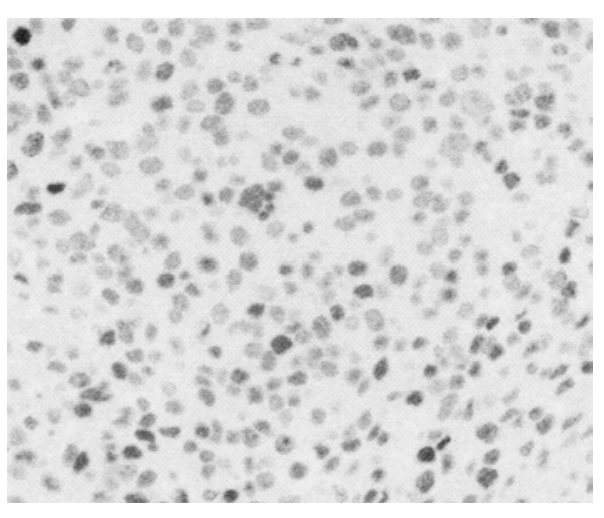

C

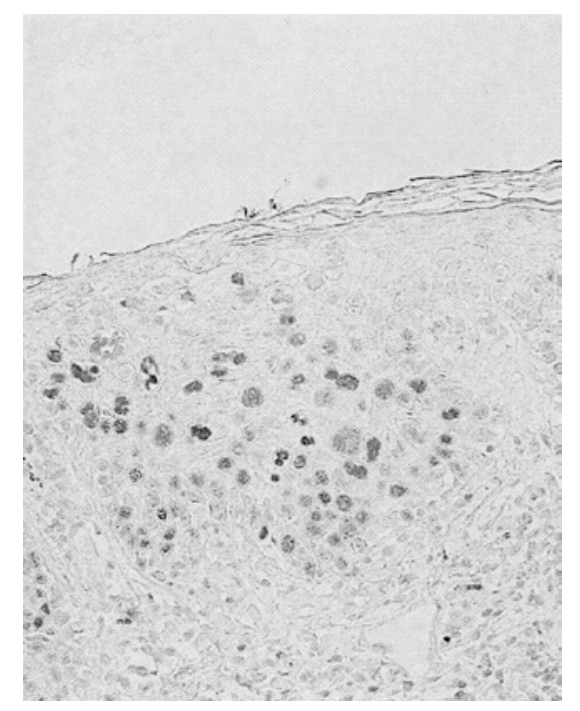

B

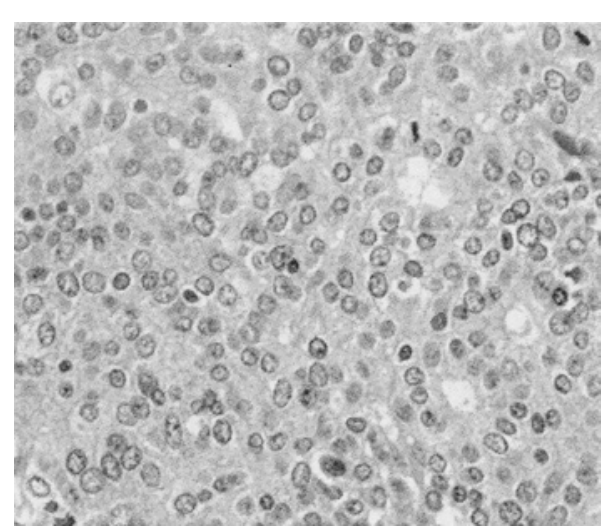

E

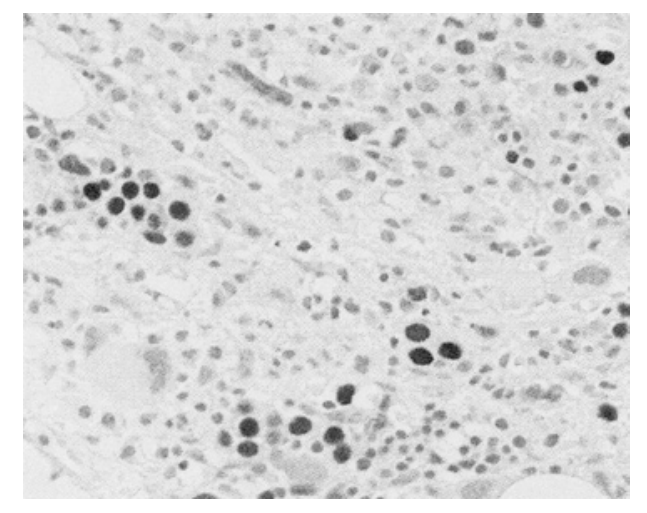

D

Figure 3. Immunohistochemistry. A-D, Immunostaining using anti-p53 antibody (×200, DO-7; DAKO, Tokyo) A, normal skin; B, Bowen's disease; C, ovarian cancer; D, bone marrow. Bowen's disease lesion, ovarian cancer, and bone marrow cells showed accumulation of $\mathbf{p 5 3}$ in the nuclei. $\mathrm{E}, \mathrm{H}-\mathrm{E}$ staining of ovarian cancer $(\times 200)$.

(Fig. 2B, C). To elucidate the cause of this peak, DNA from peripheral blood, the Bowen's disease lesion and normal skin were further subjected to direct sequencing of TP53, and it was revealed that they all had a missense mutation in codon 175 ( $\mathrm{G}$ to $\mathrm{A}$ ) such that arginine had been replaced with histidine (Fig. 2E, F, G). There also was a normal sequence at codon 175. Because there were mutations not only in the peripheral blood and in the Bowen's disease lesion but also in normal skin, and because all these mutations existed in the same codon and in the same manner, it is speculated that our patient carries a TP53 germline mutation. Previously resected ovarian cancer tissue was subjected to immunostaining using the anti-p53 antibody, revealing an accumulation of p53 in the nuclei (Fig. 3C). The same study of bone marrow biopsy specimen also revealed that about $20 \%$ of the cells had p53 nuclear accumulation (Fig. 3D). After informed consent, lymphocyte DNA from her two sons was examined for
TP53 mutation, but none was found. We were unable to search for the germline TP53 mutation among her relatives with cancer as they were deceased, and we could not obtain informed consent to examine TP53 of relatives without cancer. For these reasons, it was unclear whether she was a proband or her TP53 mutation was inherited.

\section{Discussion}

We have encountered a case of myelodysplastic syndrome carrying TP53 germline mutation. TP53 is a tumor suppressor gene, and is now known to be the most frequently altered gene in human tumors $(13,14)$. Codon 245, 248, 249, 273 and 175, identified in the present case, are hotspots of TP53 mutations, representing $20 \%$ of all known mutations (15). According to a database of germline p53 mutations in cancer-prone families, 49 cases with $\mathrm{R} 175 \mathrm{H}$ have been reported 
(16); 34 cases were from Li-Fraumeni families, and 15 cases were from others, including 3 cases without cancer. Among these cases were three cases of acute lymphoblastic leukemia, but no other hematological malignancies have been reported.

It is well known fact that some cases of MDS and acute myeloid leukemia arise in patients previously treated with irradiation or chemotherapy for other malignancies $(8,17)$. Therapy related leukemia/ MDS are classified into two categories (18). The first type generally presents 5 to 15 years after exposure to alkylating agents and has a high incidence of chromosomal abnormalities, particularly in chromosomes 5 and 7. In addition, patients usually develop trilineage dysplasia and severe fibrosis of the bone marrow. The second type of therapy related leukemia/ MDS is associated with the use of topoisomerase II inhibitors, and they show balanced chromosomal aberrations. Patients in this category have a more favorable response to chemotherapy than those in the former group. The present case developed MDS 5 years after chemotherapy against ovarian cancer. G-banding of the MDS clone showed deletion of chromosomes 5, 6, 7, and 17. Karyotype and clinical features indicated that our case belonged to the first type of t-MDS. It is speculated that loss of p53 functions due to TP53 germline mutation with the loss of a wild type allele, most likely induced by the chemotherapy administered for her ovarian cancer, caused the development of MDS in our case.

Bowen's disease is one of the precursors of nonmelanoma skin cancer (19), and it has been reported that 31 to $47 \%$ of Bowen's disease cases show TP53 mutations (911). The results of immunostaining in our case suggested that p53 promotes the development of Bowen's disease as p53 had accumulated in the nuclei of the lesion but not in normal skin. However, there was no additional abnormal peak in the SSCP of exons 5-8 in the Bowen's disease lesion compared to that of the normal skin. From these results, we speculate that there may be an additional alteration of TP53 outside exons $5-8$, or there may be an additional factor influencing p53 in the Bowen's disease lesion.

It has been reported that approximately $50 \%$ of ovarian cancers show TP53 mutation and have a critical role in its pathogenesis (20). As our patient's ovarian cancer tissue showed p53 accumulation, TP53 mutation may have been involved in the pathogenesis of her ovarian cancer, and it is acceptable from the result that she had already had a TP53 germline mutation before the development of ovarian cancer. We could not determine if there were any factors influencing the development of ovarian cancer other than p53 in our case.

The present case responded only to prednisolone treatment. As cyclosporine A did not have any response in our case, it is speculated that not the immunosuppressive but the anti-tumor or anti-fibrogenic effects of steroid therapy induced the hematologic response. As there have been similar reports which show steroid therapy improving myelofibrosis (21-24), it may be a viable alternative for patients with myelofibrosis who cannot undergo stem cell transplantation.

Acknowledgements: The authors thank Mr. Kevin Litton (Bachelor of Arts in English) for editorial assistance.

\section{References}

1) Fenaux P. Chromosome and molecular abnormalities in myelodysplastic syndromes. Int J Hematol 73: 429-437, 2001.

2) Sugimoto K, Hirano N, Toyoshima H, et al. Mutations of the p53 gene in myelodysplastic syndrome (MDS) and MDS-derived leukemia. Blood 81: 3022-3326, 1993.

3) Kaneko H, Misawa S, Horiike S, Nakai H, Kashima K. TP53 mutations emerge at early phase of myelodysplastic syndrome and are associated with complex chromosomal abnormalities. Blood 85: 2189-2193, 1995.

4) Misawa S, Horiike S. TP53 mutations in myelodysplastic syndrome. Leuk Lymphoma 23: 417-422, 1996.

5) Fenaux P, Jonveaux P, Quiquandon I, et al. P53 gene mutations in acute myeloid leukemia with 17p monosomy. Blood 78: 1652-1657, 1991.

6) Denzlinger C, Bowen D, Benz D, Gelly K, Brugger W, Kanz L. Lowdose melphalan induces favourable responses in elderly patients with high-risk myelodysplastic syndromes or secondary acute myeloid leukaemia. Br J Haematol 108: 93-95, 2000.

7) Kita-Sasai $Y$, Horiike S, Misawa S, et al. International prognostic scoring system and TP53 mutations are independent prognostic indicators for patients with myelodysplastic syndrome. Br J Haematol 115: 309312,2001

8) Pedersen-Bjergaard J, Pedersen M, Roulston D, Philip P. Different genetic pathways in leukemogenesis for patients presenting with therapyrelated myelodysplasia and therapy-related acute myeloid leukemia. Blood 86: 3542-3552, 1995.

9) Campbell C, Quinn AG, Ro YS, Angus B, Rees JL. p53 mutations are common and early events that precede tumor invasion in squamous cell neoplasia of the skin. J Invest Dermatol 100: 746-748, 1993.

10) Takata M, Rehman I, Rees JL. p53 mutation spectrum in Japanese Bowen's disease suggests a role for mutagens other than ultraviolet light. Int J Cancer 71: 370-372, 1997.

11) Lee HJ, Kim JS, Ha SJ, et al. p53 gene mutations in Bowen's disease in Koreans: clustering in exon 5 and multiple mutations. Cancer Lett 158: 27-33, 2000 .

12) Kawakami T, Soma Y, Mizoguchi M, Saito R. Analysis of $\mathrm{p} 53$, p21(Waf1/Cip1) and TGF-beta(3) immunohistochemical staining in Bowen's disease. Dermatology 202: 9-15, 2001.

13) Hollstein M, Sidransky D, Vogelstein B, Harris CC. p53 mutations in human cancers. Science 253: 49-53, 1991.

14) Levine AJ, Momand J, Finlay CA. The p53 tumour suppressor gene. Nature 351: 453-456, 1991.

15) Hainaut P, Soussi T, Shomer B, et al. Database of p53 gene somatic mutations in human tumors and cell lines: updated compilation and future prospects. Nucleic Acids Res 25: 151-157, 1997.

16) Sedlacek Z, Kodet R, Poustka A, Goetz P. A database of germline p53 mutations in cancer-prone families. Nucleic Acids Res 26: 214-215, 1998.

17) Takeyama K, Seto M, Uike N, et al. Therapy-related leukemia and myelodysplastic syndrome: A large-scale Japanese study of clinical and cytogenetic features as well as prognostic factors. Int J Hematol 71: 144-152, 2000.

18) Bennett JM, Kouides PA, Forman SJ. The myelodysplastic syndromes: Morphology, risk assesment, and clinical management (2002). Int J Hematol 76 (suppl 2): 228-238, 2002.

19) Sober AJ, Burstein JM. Precursors to skin cancer. Cancer 75 (Suppl 2): 645-650, 1995.

20) Schuijer M, Berns EM. TP53 and ovarian cancer. Hum Mutat 21: 285291, 2003. 


\section{MDS Associated with Bowen's Disease and Ovarian Cancer}

21) Ozsoylu S. High-dose intravenous methylprednisolone for idiopathic myelofibrosis. Lancet 1: 766, 1988.

22) Tanosaki R, Uchida $H$, Masuda $Y$, et al. Improvement of bone marrow hematopoiesis in idiopathic myelofibrosis with prednisolone. Rinsho Ketsueki (Jpn J Clin Hematol) 33: 256-258, 1992 (in Japanese).

23) Jack FR, Smith SR, Saunders PW. Idiopathic myelofibrosis: anaemia may respond to low-dose dexamethasone. Br J Haematol 87: 877-878, 1994.

24) Yumoto Y, Okuda T, Yoshida Y. Primary myelofibrosis with positive coombs' test responding to prednisolone. Rinsho Ketsueki (Jpn J Clin Hematol) 37: 255-259, 1996 (in Japanese). 